\title{
Transforming Carcinogenesis is Essential Substrate Accumulation/Interactivity Dimension in Ubiquitin- Proteasomal Dysfunction
}

\author{
lawrence M Agius* \\ Department of Pathology, University of Malta Medical School, Europe
}

Received: 棒 June 07, 2018; Published: 非July 05, 2018

*Corresponding author: lawrence M Agius, Department of Pathology, University of Malta Medical School , Europe, Tel: 356-21451752;

Email: lawrence.agius@um.edu.mt

\begin{abstract}
The constitutive susceptibilities to malignant transformation are well illustrated by the evolving dimensions of a dysfunctional ubiquitin proteasomal participation in generative reformulation of carcinogenetic pathways of interactivity. Further considerations indicate the transformation as derivative dysfunctional ubiquitin proteasomal system for further transformation. The multilayered complexities of such changes attest for redistribution of protein intermediates within various cellular subcompartments as signified by endosomal and nuclear systems of cooperative interchange. Dynamics formulation is itself a parametric measurement in substitution modelling of substrates as indeed projected within system profile for further change.Theincremental disjunction of such formulasareproposed alternative systems in further parahomeostatic dysregulation in carcinogenesis as a primary generative induction as signified by further developmental adaptation of such systems as DNA repair pathways.
\end{abstract}

Keywords: Carcinogenetic; Parahomeostatic; Proteasomal System; Leukemogenesis; Myeloid; Homeostasis; Endosomes; Ubiquitin; Tumor; Phosphorylation

\section{Introduction}

The ubiquitin proteasomal system is essential requisite schematic machinery in the operative dimensional functioning of whole pathway operability in homeostasis.Such system is hence potential dysfunctioning of the various mechanisms ranging from receptor turnover to endosomes, cell cycle dynamics, DNA repair processes. Ubiquilin4, a member of the UbL-UBA protein family, serves as an adaptor in the degradation of specific substrates via the proteasomal pathway and exerts anti-tumor potential in gastric cancer by p53dependent and p53independent regulation of p21 [1]. Ubiquitinspecific protease 15 is essential asanhuman papillomavirus16 E6interacting protein [2]. The ubiquitin and ubiquitinlike intermediates are hence a core system that operates also in induced dysfunction of cell disease and cancer.USP22 deficiency leads to myeloid leukemogenesis upon activation of oncogenic Kras activation through a PU1 dependent mechanism [3]. It is relative to such considerations that intense investigation has been devoted to modulate and suppress the dysfunctional proteasomal pathways to suppress carcinogenesis.

\section{Dynamics of Operability}

Dynamics of operability of the ubiquitin proteasomal system exhibit exquisite specificity for protein molecular targeting and hence are ideally constituted in the face of a broad range of protein substrates through posttranslational modification of numerous protein moieties. In such manner, evolving pathways can potentially incriminate the endless formation of monoubiquitylation conjugates and also polymeric chains of ubiquitin in the formulation of dysfunctional regulation of cellular pathways that otherwise are of significance in homeostatic control. HERC proteins exert ubiquitin ligase activity and are widely involved in various cancers [4]. Increments of operability include multilayered complexity in the system profile dysfunctions in cancer as wellillustrated by the various developmental pathways that operate in progression of carcinogenesis. Ubiquilin4 regulates the degradation of many proteins implicated and may induce cell cycle arrest and apoptosis via ERK activation and upregulation of cyclin D1 [5]. Carcinogenesis is a parahomeostatic series of mechanistic pathways that operates in terms of adaptor systems and as dysfunction adaptation to dysregulatory lesions within cellular pathways. Deubiquitinylase USP47 promotes RelA phosphorylation and survival ingastric cancer cells [6]. The incremental dimensions for polyubiquitylation include the creation also of polymer homotypic and also heterotypic ubiquitin chains that compete with ubiquitin and ubiquitinlikesystems for Glycineinduced attachments to the Lysine moiety of substrate proteins. SUMOylation is covalently modified on lysine residues of a target protein and regulates transcription, cell cycle, DNA repair and innate immunity [7]. 


\section{Complexity}

Many layers of complexity characterize single and multiple rounds of ubiquitylation in a manner that allows for crosstalk to ubiquitin like modulators such as NEDDylation and SUMOlylation of various protein substrates.Cullin Ring Ligases in particular are implicated in such crosstalk in a manner that implicates promiscuous interactivities of whole protein substrate pathways leading to carcinogenesis. STXBP4 regulates anaphase promoting complex or cyclostomes mediated p63 turnover and drives squamous cell carcinogenesis [8]. The development of parahomeostatic dysregulation is hence a prerequisite in the formulation of dimensional adaptation to cellular dysfunction. E3 ubiquitin ligase RAD18 and its downstream effectors of the Yfamily DNA polymerases participate in different phases of multistep carcinogenesis [9]. Developmental disorders implicate and further enhance the ubiquitin-proteasomal system within the various dimensions for complexity disorders. Histone chaperone ASF1A is physically associated with USP52 which in turn acts as a deubiquitinase in chromatin assembly with a potential role in breast carcinogenesis [10]. The ubiquitinspecific protease USP28 deficiency promotes breast and liver carcinogenesis as well as tumor angiogenesis in a HIF-independent manner [11]. The performance of incremental dysfunctionality is proof of principle towards the changes in profile interactivity of protein substrates that may compete with ubiquitin in the stabilization of suppressors and oncogenes such as p53 and HDM2 formulas. SUMO catalytic cycle includes maturation, activation, conjugation, ligation and demodification and is widely involved in carcinogenesis, DNA damage response, cancer cell proliferation, metastasis and apoptosis [12].

\section{Derivative Phenomena}

Derivativescope for multilayered complexities in ubiquitylation is an attempt for maladjustment in formulating the further comparative dimensions as projected by protein substrate interactions to active sites on other protein systems within the cell. The performance co-operativity of such system pathways hence augment the range of effects of operative ubiquitylation towards such profile systems as demonstrated by multi-compartment dysfunction within cells that subsequently undergo malignant transformation. The cullin family of proteins is implicated in the ubiquitin or mediated degradation of proteins, modulating cell proliferation, cellcycle control, migration, invasion and metastasis in tumor progression [13]. Wholegenome sequencing of colorectal cancer identified amplification of Ring finger protein 6, a RINGdomain E3 ubiquitin ligase, and plays a pivotal oncogenic role in colorectal tumourigenesis [14].

System adaptation in the formulation of dysfunctional profiles in ubiquitylation and of ubiquitylation-like formulas allow for extensive impact dynamics in the non-resolution of pathway generation and progression. Processivity systems permit the modulation of injury or lesion demarcation that comes to dominate in extensive manner the protein milieu of pathway progression in parallel modetoaffect multiple parahomeostatic systems of effect and transformation. Ubiquitination and ubiquitinlike post translational modifications regulate activity and stability of oncoproteins and tumor suppressors; USP18 is the major DUB that removes IFNstimulated gene 15 from conjugated proteins [15].

\section{Maladaptation}

Intense investigations have been carried out in profile formulation and require the distinctive global dimensions of cellular dysfunction that promotes accumulation of various projected moieties in manner cooperativity when the ubiquitinproteasomal system is inhibited.Such formulas of adaptive dysfunction include gene suppressors in a manner that is modeled by stabilization of the p53. Performance increments detail the constitutive and parahomeostasis that redefine the projected dynamics of turnover of proteins in terms that signify further operativetransformation of biochemical and biophysicaldimensions of maladaptation as well exemplified by nuclear repair attempts of damage.

\section{Para Homeostasis}

Cooperative parahomeostasis is hence complexitydriven in terms of the initiation and further constitutive progression of DNA damage and as abnormal cell compartmentalization of protein moieties.The distinctive compartmentalizationofsuch protein molecules incrementallyallow for permissive reconstitution as projected within schemes for further complexity formulation.The active participation of DNA damage and the accompanying systems in DNA repair systems are further dimensionalized as well developed maladaptive system characterization and recharacterization. RINGFinger protein 6 amplification activates JAK or STAT3 pathway by modifying SHP1 ubiquitylation and associates with poor outcome in colorectal cancer [16]. The pathways that formulate DNA damage are themselves modified in terms of milieu transformation within carcinogenetic initiation and progression.Ubiquitylation pathways initiated specifically by UBA6 set a suppressive barrier against critical steps of mammary carcinogenesis such as loss of polarity, anoikis resistance and epithelial mesenchymal transition [17]. Such pathway systems perform idealistic recapitulation within the broad range for competitive protein interactivity as induced by altered ubiquitin-proteasomal function and dysfunction.

\section{Concluding Remarks}

Derivative characterizations of inhibition and transformation of interactivities of the ubiquitin or proteasome is ideal model participation in the realization of system reformulation in a manner that promotes a series of adaptive transformations consequent to accumulative dynamics with substrateenhanced inhibition of the ubiquitylation of a broad range of protein metabolism and pathway crosstalk interactivity. MARCH8 belongs to a family of membrane associated RING-CH(MARCH) ubiquitin ligases and is associated with poor prognosis in non-small cell lung cancer patients [18]. Formulas are created and these generate various maladaptive transforming influences in the system profile projection of such global participation as carcinogenesis. Projected complexity systems well demonstrate the incremental formulas as pathway cross-talk and cooperative interaction as system modulatory pathways that conceive of generative transformation to carcinogenetic pathways for further transformation. Performance utilization pathways 
induce enhanced process formulation that is mirrorimaged in the DNA damage to repair systems of adaptation and readaptation. The performance attributes for further transformation in terms of maladaptive change allow for incremental progression in cell cycling events and as selective participation that project with the onset impact of transforming parahomeostasis. Distributed compartmentalization of intermediate participants allows forcomplex microenvironmental modulation within transformed dynamics for carcinogenesis that is both adaptive and generative within the global cellular dimensions for projected complex interactivity.

\section{References}

1. Huang S, LiY, Yuan X, Zhao M, Wang J, et al. (2018) The UbL-UBA ubiquilin4 protein functions as a tumor suppressor in gastric cancer by p53-dependent and p53-independent regulation of p21. Cell Death Differ.

2. Yaginuma Y, Yoshimoto M, Tokuda A (2018) USP15 inhibits HPV16 E6 degradation and catalytically inactive USP15 has reduced inhibitory activity Acta Virol 62(2):147-156.

3. MeloCardenas J, Xu Y, Wei J, Tan C, Kong S, et al. (2018) USP22 deficiency leads to myeloid leukemia upon oncogenic Kras activation through a PU.1 dependent mechanism. Blood.

4. Mao X, Sethi G, Zhang Z, Wang Q (2018) The emerging roles of the HERC ubiquitin ligases in cancerCurr Pharm Des.

5. Huang S, Dong X, Wang J, Ding J, Li Y, et al. (2018) Overexpression of the ubiquilin-4 (UBQLN4) is associated with cell cycle arrest and apoptosis in human normal gastric epithelial cell lines GES-1 cells by activation of the ERK signaling pathway. Med Sci Monit 24: 3564-3570.

6. Naghavi L, Schwalbe M,Ghanem A, Naumann M (2018) Deubiquitinylase USP47 promotes RelA phosphorylation and survival in gastric cancer cells. Biomedicines.

7. Yang Y, Xia Z, Wang X, Zhao X, Sheng Z, et al. (2018) Smallmolecule inhibitors targeting protein SUMOylation as novel anticancer compounds. Mol Pharmacy 94(2): 885-894.

\section{ISSN: 2574-1241}

DOI: 10.26717/BJSTR.2018.06.001357

lawrence M Agius. Biomed J Sci \& Tech Res

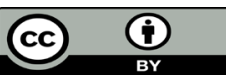

This work is licensed under Creative Commons Attribution 4.0 License

Submission Link: https://biomedres.us/submit-manuscript.php
8. Rokudai S, Li Y, Otaka Y, Fujieda M, Owens DM, et al. (2018) STXBP4 regulates APC/C-mediated p63 turnover and drives squamous cell carcinogenesis. Proc Natl Acad Sci USA 115(21): 4806-4814.

9. Yang Y, Gao Y, Ziatanou A, Tateishi S, Yurchenko V, et al. (2018) Diverse roles of RAD18 and Y-family DNA polymerases in tumorigenesis. Cell Cycle p. 1-11.

10. Yang S, Liu L, Cao C, Song N, Wang Y, Ma S, et al. (2018) USP52 acts as a deubiquitinase and promotes histone chaperone ASF1A stabilization. Nat Commun 9(1): 1285.

11. Richter K, Paarkola T, Mennerich D, Kubaichuk K, Kippari HA, et al. (2018) USP28 deficiency promotes breast and liver carcinogenesis as well as tumor angiogenesis in a HIF-independent manner. Mol Cancer Res 16(6): 1000-1012.

12. Han ZJ, Feng YH, Gu BH, Li YM, Chen H (2018) The post-translational modification, SUMOylation, and cancer. Int J Oncol 52(4): 1081-1094.

13. Michail O, Moris D, Theocharis S, Griniatsos J (2018) Cullin-1 and -2 protein expression in colorectal cancer: correlation with clinicopathological variables In Vivo 32(2): 391-396.

14. Liu L, Zhang Y, Wong CC, Zhang J, Dong Y, et al. (2018) RNF6 promotes colorectal cancer by activating the Wnt/beta-catenin pathway via ubiquitination of TLE3Cancer Res 78(8): 1958-1971.

15. Mustachio LM, Lu Y, Kawakami M, Roszik J, Fremantle SJ, et al. (2018) Evidence for the ISG15-specific deubiquitinase USP18 as an antineoplastic target. Cancer Res 78(3): 587-592.

16. Liang Q Ma D, Zhu X, Wang Z, Sun TT, et al. (2018) RING-Finger protein 6 amplification activates JAK/STAT3 pathway by modifying SHP-1 ubiquitylation and associates with poor outcome in colorectal cancer Clin Cancer Res 24(6): 1473-1485.

17. Liu X, Sun L, Gursel DB, Cheng C, Huang S, et al. (2017) The non-canonical ubiquitin activating enzyme UBA6 suppresses epithelial-mesenchymal transition of mammary epithelial cells. Oncotarget 8(50): 87480-87493.

18. Fan J, Tian L, Li M, Huang SH, Zhang J (2017) MARCH8 is associated with poor prognosis in non-small cell lung cancer patients Oncotarget 8(64): 108238-108248.

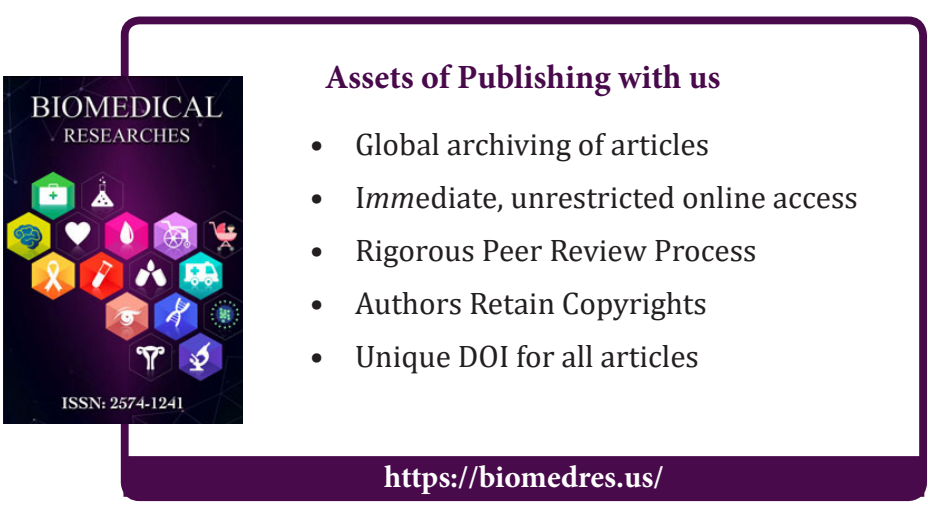

Cite this article: lawrence M A. Transforming Carcinogenesis is Essential Substrate Accumulation/Interactivity Dimension in Ubiquitin-

Proteasomal Dysfunction. Biomed J Sci\&Tech Res 6(3)- 2018. BJSTR. MS.ID.001357. DOI: 10.26717/ BJSTR.2018.06.001357. 